\title{
An economic evaluation of using raltegravir in treatment-experienced HIV-I infected patients in the UK E Elbasha ${ }^{1}$, W Dunlop*2, MA Chaudhary ${ }^{1}$ and RN Kumar ${ }^{3}$
}

Address: ${ }^{1}$ Merck and Co, Inc, North Wales, USA, ${ }^{2}$ Merck Sharpe and Dohme, Hoddesdon, UK and ${ }^{3}$ Merck and Co, Inc, Whitehouse Station, USA

* Corresponding author

from Ninth International Congress on Drug Therapy in HIV Infection

Glasgow, UK. 9-13 November 2008

Published: 10 November 2008

Journal of the International AIDS Society 2008, I I (SuppI I):P3I0 doi:I0.I I86/I758-2652-II-SI-P3I0

This abstract is available from: http://www.jiasociety.org/content/II/SI/P3 I0

(c) 2008 Elbasha et al; licensee BioMed Central Ltd.

\section{Background}

Raltegravir is the first in the new class of integrase inhibitors. In treatment-experienced patients with advanced HIV disease, raltegravir in combination with optimized background therapy (OBT) showed superior efficacy compared with placebo with OBT at week 16, 24, and 48. This research focuses on the cost-effectiveness of raltegravir from the National Health Service (NHS) perspective.

\section{Methods}

We developed a decision model to estimate the long-term (over 30 years) clinical and economic outcomes associated with using raltegravir in treatment-experienced HIV patients in the UK (UK) for 5 years using the NHS perspective. The model stratifies HIV-1 infected persons into several heath states according to their HIV-RNA level, CD4lymphocyte cell count, and history of opportunistic infections (OI). Each health state is associated with a distinct set of costs (for prophylaxis, testing, and treatment of complications) and quality of life (utility) scores. Inputs for the model were obtained from clinical trials, published studies, and database analysis. Output included number and type of OI, projected life expectancy, qualityadjusted life expectancy, lifetime direct costs, and incremental cost-effectiveness ratios (ICER). The ICERs were expressed in 2007 British pounds per quality-adjusted life year (QALY) gained ( $f / Q A L Y)$. Both future costs and QALYs were discounted to the present value at 3.5\% per year. Extensive sensitivity and subgroup analyses were conducted.

\section{Summary of results}

Projected model outcomes were generally within the range of values reported in the literature. Compared with OBT, raltegravir (as an add-on to OBT) increased discounted QALYs by 2.19 years at an additional discounted cost of $£ 35,984$, resulting in an ICER of $£ 16,431$ /QALY. The results were sensitive to changes in utility weights, treatment duration, mortality rates, health utility weights, analytic time horizon, cost of OBT, cost of raltegravir, incidence of OIs, and discount rate. However, in most scenarios assessed, the ICER was below $£ 20,000$.

\section{Conclusion}

Use of raltegravir is projected to result in substantial quality-adjusted survival benefits and is cost-effective in treatment-experienced patients in the UK when compared with current standard of care. 